\title{
Loss of miR-873 contributes to gemcitabine resistance in triple-negative breast cancer via targeting ZEB1
}

\author{
GANGYUE WANG, YI DONG, HENG LIU, NAN JI, JILEI CAO, AIHUI LIU, XIN TANG and YU REN \\ Department of Breast Surgery, Beijing Obstetrics and Gynecology Hospital, \\ Capital Medical University, Beijing 100006, P.R. China
}

Received January 7, 2018; Accepted October 25, 2018

DOI: $10.3892 /$ ol.2019.10697

\begin{abstract}
Gemcitabine-based chemotherapy is commonly applied for the treatment of breast cancer in a clinical setting. However, acquired resistance to chemotherapy primarily results in treatment failure and eventually culminates in patient mortality. Aberrant expression of microRNAs (miRNAs) has been demonstrated to be implicated in the development of chemoresistance; however, the role of miR-873 in the chemoresistance of breast cancer and its underlying mechanism have not been completely elucidated. Herein, using cell viability assays, the present study demonstrated that overexpression of miR-873 sensitized triple-negative breast cancer (TNBC) cells (MDA-MB-231 and BT549) towards gemcitabine treatment, while inhibition of miR-873 promoted resistance of TNBC cells to gemcitabine exposure. The 3' untranslated region of zinc finger E-box binding homeobox 1 (ZEB1) was predicted as a candidate target of miR-873, and the regulatory association between ZEB1 and miR-873 was validated with a dual luciferase assay. Reverse transcription-quantitative polymerase chain reaction and western blot analysis confirmed that miR-873 mimics reduced ZEB1 at mRNA and protein levels in MDA-MB-231 and BT549 cells. As ZEB1 was previously reported to interact with Yes associated protein (YAP) to promote cancer progression. The present study observed that miR-873 overexpression decreased the expression of YAP target genes AXL receptor tyrosine kinase, connective tissue growth factor and cysteine rich angiogenic inducer 61 at mRNA and protein levels. Additionally, elevation of the ZEB1 level and reduction of the miR-873 level were detected in gemcitabine-resistant MDA-MB-231 (MDA-MB-231GEMr) cells, which were accompanied with stronger proliferative ability, compared with parental cells. Overexpression of miR-873 or ZEB1
\end{abstract}

Correspondence to: Dr Gangyue Wang, Department of Breast Surgery, Beijing Obstetrics and Gynecology Hospital, Capital Medical University, 17 Qihe lou Road, Beijing 100006, P.R. China E-mail: wanggangyuecmu@sina.com

Key words: microRNA-873, zinc finger E-box binding homeobox 1, gemcitabine resistance, triple-negative breast cancer knockdown reversed chemoresistance of MDA-MB-231GEMr cells by inducing a notable cell growth arrest upon gemcitabine exposure. In conclusion, the data obtained by the present study demonstrated that the decrease of miR- 873 promoted the development of gemcitabine resistance in TNBC via elevation of ZEB1 expression, which indicated that miR-873 may be a promising predictor for gemcitabine sensitivity in patients with TNBC.

\section{Introduction}

Accounting for $15 \%$ of breast cancer cases worldwide, triple-negative breast cancer (TNBC) is an aggressive subtype of breast cancer, which is characterized by the lack of estrogen receptor, progesterone receptor and human epidermal growth factor receptor 2 (HER2) (1). Despite high sensitivity towards chemotherapy, the overall survival rate of patients with TNBC remains poor due to the frequently occurring relapse (2).

Gemcitabine is a chemotherapy agent, which is derived from deoxycytidine, and is commonly used for the treatment of patients with breast cancer (3). Gemcitabine requires intracellular transport and induces cell cycle arrest via incorporating into DNA or inhibition of ribonucleotide reductase (4). Although patients respond to gemcitabine at the beginning of treatment, numerous patients eventually develop secondary resistance, which may result in patient mortality (5). Thus, further investigation regarding the molecular mechanism of gemcitabine resistance would facilitate the development of novel therapeutic approaches and improve patient outcomes.

microRNAs (miRNAs) are small, non-coding, single strand RNAs with a length of $\sim 20$ nucleotides (6). Through binding to 3' untranslated region (UTR) of target gene mRNAs, miRNAs directly inhibit gene expression at the post-transcriptional level (7). Deregulation of numerous miRNAs has been determined to be associated with the chemotherapy resistance of breast cancer (8). For example, miR-105 and miR-93-3p can target secreted frizzle related protein 1 to activate Wnt signaling, which leads to chemoresistance in TNBC cells (9). miR-873 was reported to be downregulated in numerous cancer types, including breast cancer $(10,11)$. Previous studies indicated that miR-873 may function as a sensitizer of cancer cells towards chemotherapeutic agents $(12,13)$. However, whether and how miR-873 influences chemosensitivity in TNBC remains unclear. 
Zinc finger E-box binding homeobox 1 (ZEB1) is a transcription factor that is well-known for its role as an epithelial-mesenchymal transition (EMT) inducer (14). Through the induction of EMT, ZEB1 promotes cancer cell dissociation, resulting in metastasis and poor prognosis of patients with cancer $(15,16)$. Upregulation of ZEB1 was identified in TNBC, compared with other breast cancer subtypes (17). Notably, ZEB1 elevation was detected in circulating breast cancer cells, which were characterized with cancer stem cell traits including self-renewal and strong metastatic potential (18). Mechanistically, ZEB1 coordinated with Hippo-pathway effector Yes associated protein (YAP) to maintain cell stemness and promote metastasis (19). Via maintenance of cancer cell stemness, ZEB1 has been identified to contribute to the development of cancer cell chemoresistance (14).

In the present study, the role of miR-873 in the regulation of chemosensitivity in TNBC was demonstrated, and it was revealed that miR-873 downregulation led to gemcitabine resistance of TNBC cells via regulation of ZEB1. The results depicted a novel role of miR-873 in mediating gemcitabine sensitivity in TNBC cells, indicating that the expression of miR-873 may serve as a predictor for gemcitabine sensitivity of patients with TNBC.

\section{Materials and methods}

Cell culture and agents. The human kidney cell line 293 and human TNBC cell lines (MDA-MB-231 and BT549) were purchased from American Type Culture Collection (Manassas, MA, USA). All cell lines were cultured in Dulbecco's minimum essential medium (DMEM; Gibco; Thermo Fisher Scientific, Inc., Waltham, MA, USA) supplemented with $10 \%$ fetal bovine serum (FBS; Hyclone; GE Healthcare Life Sciences, Logan UT, USA) in an incubator containing $5 \% \mathrm{CO}_{2}$ at $37^{\circ} \mathrm{C}$.

The gemcitabine resistant MDA-MB-231 cell line (MDA-MB-231GEMr) was generated by continuous exposure of MDA-MB-231 to increasing concentrations (0.1-15 nM) of gemcitabine (Sigma-Aldrich, Merck KGaA, Darmstadt, Germany) for 12 months at $37^{\circ} \mathrm{C}$, according to the method of a precious report (20). The MDA-MB-231GEMr cells were cultured in DMEM with 10\% FBS and $15 \mathrm{nM}$ gemcitabine, and incubated in an incubator with $5 \% \mathrm{CO}_{2}$ at $37^{\circ} \mathrm{C}$ prior to experiments. Prior to additional experiments, $1 \times 10^{6}$ MDA-MB-231GEMr cell passage in DMEM without gemcitabine was conducted twice.

Overexpression and downregulation of $m i R-873$. $\mathrm{miR}-873$ mimics, miR-negative control (miR-NC) mimics, miR-873 inhibitor and miR-NC inhibitor were obtained from Chang Jing Bio-Tech, Ltd. (Changsha, China). For overexpression or downregulation of miR-873, $100 \mathrm{nM}$ miR-873 mimic or miR-873 inhibitor was transfected into MDA-MB-231 or BT549 cells with Lipofectamine ${ }^{\circledR} 3000$ (Invitrogen; Thermo Fisher Scientific, Inc.), according to manufacturer's protocol. Following $48 \mathrm{~h}$, the cells were subjected to the further experiments. The sequences are listed in Table I.

Silencing of ZEB1 in TNBC cells. Control small interfering RNA (siRNA), ZEB1 siRNA1 and ZEB1 siRNA2 were obtained from Chang Jing Bio-Tech, Ltd.. For silencing of
ZEB1 expression, 50 nM ZEB1 siRNA1 or ZEB1 siRNA2 were transfected into MDA-MB-231GEMr cells using Lipofectamine ${ }^{\circledR}$ RNAiMax (Invitrogen; Thermo Fisher Scientific). Following $48 \mathrm{~h}$, the cells were subjected to further experiments. The sequences are listed in Table II.

Western blot analysis. Antibodies for E-cadherin (catalog no. 14472; 1:2,000), AXL receptor tyrosine kinase (AXL; catalog no. 8661; 1:2,000), connective tissue growth factor (CTGF; catalog no. 86641; 1:2,000), cysteine rich angiogenic inducer 61 (CYR61; catalog no. 14479; 1:2,000) and ZEB1 (catalog no. 3396; 1:2,000) were purchased from Cell Signaling Technology (Cell Signaling Technology, Inc., Danvers, MA, USA). The GAPDH antibody (catalog no. SAB1403850; 1:10,000) was purchased from Sigma-Aldrich (Merck $\mathrm{KGaA}$ ). Horseradish peroxidase-conjugated secondary antibodies against rabbit (catalog no. SA00001-2; 1:10,000) and mouse (catalog no. SA00001-1; 1:10,000) were obtained from ProteinTech Group, Inc. (Chicago, IL, USA). Protein lysates were prepared with radioimmunoprecipitation assay lysis buffer (Beyotime Institute of Biotechnology, Haimen, China). The concentration of lysates was determined with a BCA Protein assay kit (Pierce; Thermo Fisher Scientific, Inc.). Protein extracts $(20 \mu \mathrm{g})$ were then separated on an $8 \%$ SDS-PAGE gel and transferred to polyvinylidene fluoride membrane. The membrane was then blocked with 5\% non-fat milk at room temperature for $0.5 \mathrm{~h}$, followed by incubation of indicated primary antibodies overnight at $4^{\circ} \mathrm{C}$. The following day, the membrane was washed three times with TBS with $0.1 \%$ Tween 20 (TBST) at room temperature for 10 mins and then incubated with secondary antibodies for $1 \mathrm{~h}$ at room temperature. Subsequently, the membrane was washed with TBST (0.1\% Tween 20) at room temperature for 10 mins (three times), developed with enhanced chemiluminescence detection agent (Thermo Fisher Scientific, Inc.) and visualized on ImageQuant TL version 1.1.0.1 (GE Healthcare, Chicago, IL, USA).

RNA extraction and reverse transcription-quantitative polymerase chain reaction ( $R T-q P C R)$. The extraction of total RNA from 293, MDA-MB-231 and BT549 cells were achieved using a miRNeasy Mini kit (Qiagen GmbH, Hilden, Germany), according to the manufacturer's protocol. The synthesis of first strand cDNA was conducted using a M-MLV kit (Thermo Fisher Scientific, Inc.). RT-qPCR was conducted to analyze specific gene levels on CFX96 (Bio-Rad Laboratories, Inc., Hercules, CA, USA) using SYBR ${ }^{\circledR}$ Premix Ex Taq (Takara Bio, Inc., Otsu, Japan). The thermocycling conditions were as follows: Pre-denaturing at $95^{\circ} \mathrm{C}$ for $30 \mathrm{sec}$, denaturing at $95^{\circ} \mathrm{C}$ for $30 \mathrm{sec}$, and 35 cycles of annealing and elongation at $60^{\circ} \mathrm{C}$ for $30 \mathrm{sec}$. GAPDH and U6 served as internal controls for mRNA and miRNA, respectively. The relative expression levels of indicated genes were calculated using the $2^{-\Delta \Delta \mathrm{Cq}}$ method (21). The primer sequences are listed in Table III.

Cell viability assay. The cell viability was detected using Cell Counting kit-8 (CCK-8; Dojindo Molecular Technologies, Inc., Kumamoto, Japan), according to the manufacturer's protocol. Briefly, 1,000 MDA-MB-231 or BT549 cells/well were seeded in 96-well plates. Following the transfection of miR-873 mimics or miR-NC mimics for $48 \mathrm{~h}, 10 \mu \mathrm{l}$ CCK- 8 solution 
Table I. Sequences of miR mimics and miR inhibitors.

\begin{tabular}{ll}
\hline Name & \multicolumn{1}{c}{ Sequence } \\
\hline miR-873 mimic & 5'-GCAGGAACUUGUGAGUCUCC \\
& UTT-3' \\
miR-NC mimic & 5'-UCGCUUGGUGCAGGUCGGGA \\
& ATT-3' \\
miR-873 inhibitor & 5'-AGGAGACUCACAAGUUCC \\
& UGCTT-3' \\
miR-NC inhibitor & 5'-UUCUCCGAACGUGUCACGUTT-3'
\end{tabular}

NC, negative control; miR, microRNA.

Table II. Sequences of control siRNA and ZEB siRNAs.

\begin{tabular}{lc}
\hline Name & Sequence \\
\hline Control siRNA & 5'-UUCUCCGAACGUGUCACGUTT-3' \\
ZEB1 siRNA1 & 5'-GUCGCUACAAACAGUUGUATT-3' \\
ZEB1 siRNA2 & 5'-CCUAGUCAGCCACCUUUAATT-3' \\
\hline
\end{tabular}

ZEB1, zinc finger E-box binding homeobox 1; siRNA, small interfering RNA.

Table III. Sequences of primers for reverse transcription-quantitative polymerase chain reaction.

\begin{tabular}{ll}
\hline Name & \multicolumn{1}{c}{ Sequence } \\
\hline Stem loop primer & 5'-CTCAACTGGTGTCGTGGAGTCG \\
& GCAATTCAGTTGAGAGGAGA-3' \\
miR-873-forward & 5'-TCGGCAGGGCAGGAACTTGTGA-3' \\
miR-873-reverse & 5'-CTCAACTGGTGTCGTGGA-3' \\
U6-forward & 5'-CTCGCTTCGGCAGCACA-3' \\
U6-reverse & 5'-AACGCTTCACGAATTTGCGT-3' \\
AXL-forward & 5'-GTGGGCAACCCAGGGAATATC-3' \\
AXL-reverse & 5'-GTACTGTCCCGTGTCGGAAAG-3' \\
CTGF-forward & 5'-CAGCATGGACGTTCGTCTG-3' \\
CTGF-reverse & 5'-AACCACGGTTTGGTCCTTGG-3' \\
CYR61-forward & 5'-CTCGCCTTAGTCGTCACCC-3' \\
CYR61-reverse & 5'-CGCCGAAGTTGCATTCCAG-3' \\
ZEB1-forward & 5'-GATGATGAATGCGAGTCAGATGC-3' \\
ZEB1-reverse & 5'-ACAGCAGTGTCTTGTTGTTGT-3' \\
GAPDH-forward & 5'-CCTGCACCACCAACTGCTTA-3' \\
GAPDH-reverse & 5'-GGCCATCCACAGTCTTCTGAG-3'
\end{tabular}

miR, microRNA; AXL, AXL receptor tyrosine kinase; CTGF, connective tissue growth factor; CYR61, cysteine rich angiogenic inducer 61; ZEB1, zinc finger E-box binding homeobox 1 .

was added into each well and incubated for $1 \mathrm{~h}$ in an incubator with $5 \% \mathrm{CO}_{2}$ at $37^{\circ} \mathrm{C}$. Following incubation the medium was transferred into another 96-well plate and the absorbance at
$450 \mathrm{~nm}$ of each well was detected using a microplate reader (Bio-Rad Laboratories, Inc.).

Dual luciferase assay. Prediction of the putative binding site between ZEB1 3'UTR and miR-873 was achieved using TargetScan software version 7.2 (http://www.targetscan. org/vert_72/). The cDNA was prepared by RNA extraction using TRIzol ${ }^{\circledR}$ reagent (Invitrogen; Thermo Fisher Scientific, Inc.) and reverse transcribed into cDNA with RevertAid First Strand cDNA Synthesis kit (Thermo Fisher Scientific, Inc.). The 3'UTR of ZEB1 mRNA was amplified from cDNA of 293 cells and was inserted into pGL3 plasmid (Promega Corporation, Madison, WI, USA) to construct pGL3-ZEB1 3'UTR-wild type (WT). pGL3-ZEB1 3'UTR-mutant (Mut) with mutation of predicted miR-873 binding sites was constructed by site mutation of pGL3-ZEB1 3'UTR-WT using a QuikChange Site-directed Mutagenesis kit (Agilent Technologies, Inc., Santa Clara, CA, USA). For dual luciferase assay, 293 cells were transfected with pGL3-ZEB1 3'UTR-WT or pGL3-ZEB1 3'UTR-Mut accompanied with miR-873 mimics or miR-negative control (NC) mimics and an internal control Renilla plasmid with Lipofectamine ${ }^{\circledR} 3000$ (Invitrogen; Thermo Fisher Scientific, Inc.). The firefly luciferase activity was firstly normalized to Renilla luciferase activity, followed by normalization to the control group. At $24 \mathrm{~h}$ post-transfection, the relative luciferase activity of each well was detected using a Dual Luciferase Reporter assay kit (Promega Corporation), according to the manufacturer's protocol.

Statistical analysis. All data were calculated and analyzed using GraphPad Prism 5.0 (GraphPad Software, Inc., La Jolla, CA, USA) and presented as mean \pm standard deviation. The differences between two groups were compared with an unpaired Student's t-test. The differences among three groups were analyzed using two-way analysis of variance followed by Newman-Keuls post-hoc analysis. $\mathrm{P}<0.05$ was considered to indicate a statistically significant difference significance. All experiments were repeated a minimum of three times.

\section{Results}

miR-873 is negatively associated with gemcitabine sensitivity in TNBC cells. To investigate the role of miR-873 in mediating gemcitabine sensitivity in TNBC cells, the present study examined the gemcitabine sensitivity of MDA-MB-231 cells and BT549 cells following miR-873 elevation and downregulation via transfection of miR-873 mimics or inhibitors, respectively. Following overexpression of miR-873 (Fig. 1A), MDA-MB-231 cells and BT549 cells became increasingly sensitive towards increasing concentrations of gemcitabine (0-8 nM), compared with their corresponding control groups (Fig. 1B-C). Conversely, antagonist of miR-873 (Fig. 1D) caused MDA-MB-231 cells and BT549 cells to be more insensitive upon gemcitabine exposure, compared with cells transfected with miR-NC inhibitor (Fig. 1E-F). These data indicate that miR-873 could mediate gemcitabine sensitivity in TNBC cells.

miR-873 directly represses ZEBI expression via binding to its 3'UTR. Using TargetScan, miR-873 was predicted to bind to 3'UTR of ZEB1 mRNA (Fig. 2A). To confirm the 
A

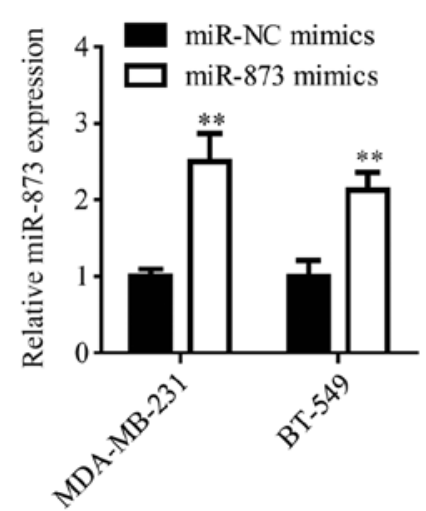

D

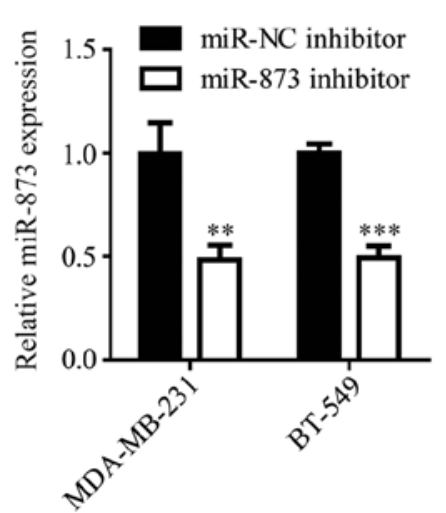

B

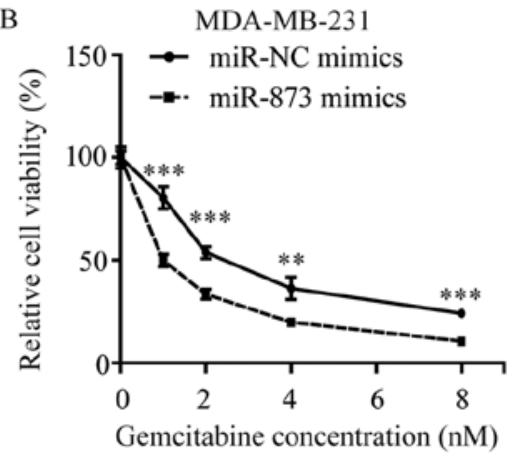

E

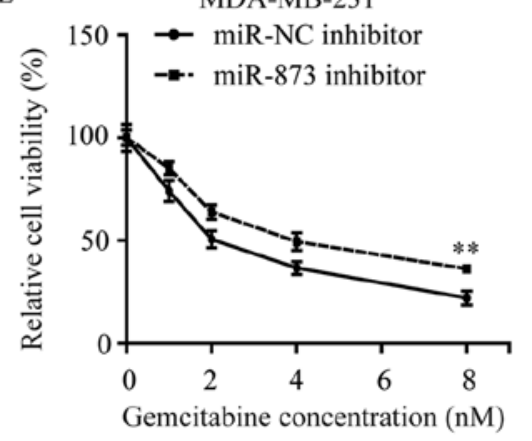

C

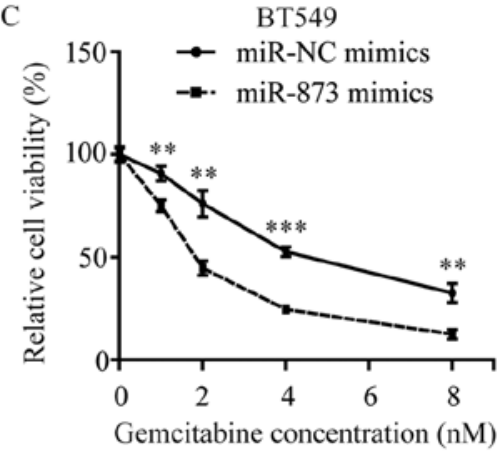

$\mathrm{F}$

BT549

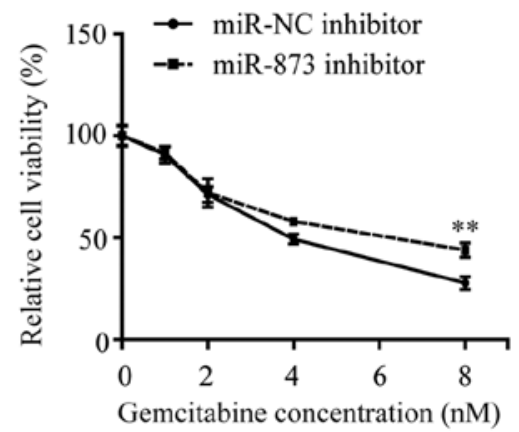

Figure 1. miR-873 mediates gemcitabine sensitivity in triple-negative breast cancer cells. (A) Transfection of miR-873 mimics elevated miR-873 level in MDA-MB-231 and BT549 cells. ${ }^{* *} \mathrm{P}<0.01$ vs. miR-NC mimics. Overexpression of miR-873 by transfection of miR-873 mimics sensitized (B) MDA-MB-231 cells and (C) BT549 cells towards gemcitabine $(0-8 \mathrm{nM}){ }^{* *} \mathrm{P}<0.01$ and ${ }^{* * *} \mathrm{P}<0.0001$ vs. miR-NC mimics. (D) Transfection of the miR-873 inhibitor reduced miR-873 levels in MDA-MB-231 and BT549 cells. ${ }^{* *} \mathrm{P}<0.01$ and ${ }^{* * *} \mathrm{P}<0.0001$ vs. miR-NC inhibitor. Downregulation of miR-873 by transfection of miR-873 inhibitor caused (E) MDA-MB-231 and (F) BT549 cells to become more insensitive towards gemcitabine (0-8 nM). ${ }^{* *} \mathrm{P}<0.01 \mathrm{vs}$. miR-NC inhibitor. NC, negative control; miR, microRNA.

regulatory association between miR-873 and 3'UTR of ZEB1, a dual luciferase assay was performed. miR-873 mimics were demonstrated to significantly downregulate the relative luciferase activity of ZEB1 3'UTR-WT but not ZEB1 3'UTR-Mut (Fig. 2B). This result validated ZEB1 as a direct target of miR-873.

miR-873 regulates $Z E B 1$ and its target genes in TNBC cells. To investigate whether miR-873 regulated ZEB1 in TNBC cells, the present study detected ZEB1 mRNA levels following miR-873 overexpression in MDA-MB-231 and BT549 cells. As illustrated in Fig. 3A, overexpression of miR-873 significantly decreased the ZEB1 mRNA level in MDA-MB-231 and BT549 cells. ZEB1 was determined to be a classic transcription suppressor and functions as an EMT inducer via repression of E-cadherin expression (22). Consistently, in MDA-MB-231 and BT549 cells, miR-873 mimics markedly decreased ZEB1 protein levels and upregulated E-cadherin protein levels (Fig. 3B and C). A recent study demonstrated that ZEB1 functions as a transcription activator through interacting with YAP1 (19). Transfection of miR-873 mimics predominantly decreased YAP target genes (AXL, CTGF and CYR61) at mRNA and protein levels in MDA-MB-231 and BT549 cells (Fig. 3D-G). These data support the notion that miR-873 regulates the target genes of ZEB1 in TNBC cells.

\section{A ZEB1 3'UTR 5'AUUUUGUGCCAAUUUGUUCCUGU 3' miR-873 3' UCCUCUGAGUGUUCAAGGACG 5'}

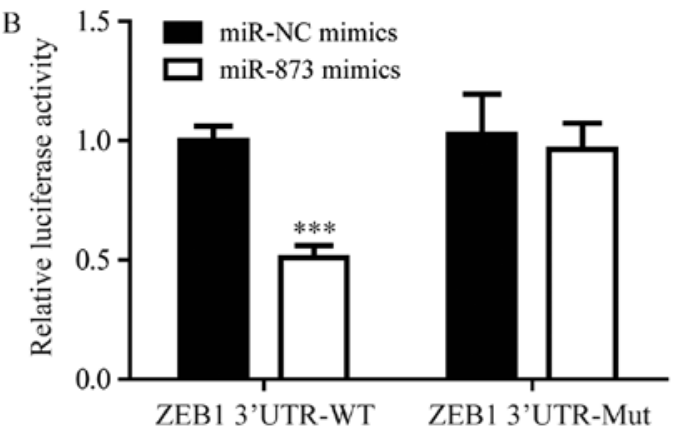

Figure 2. miR-873 could directly bind to 3'UTR of ZEB1 mRNA. (A) Sequence alignment of miR-873 and potential binding sites on 3'UTR of ZEB1 mRNA were presented. (B) Transfection of miR-873 mimics, but not miR-NC mimics, reduced the luciferase activity of 293 cells transfected with pGL3-ZEB1 3'UTR-WT. ${ }^{* * *} \mathrm{P}<0.0001$ vs. miR-NC mimics. UTR, untranslated region; ZEB1, zinc finger E-box binding homeobox 1; WT, wild type; Mut, mutant; miR, microRNA.

Aberrant expression of $m i R-873$ and ZEBI in gemcitabine-resistant MDA-MB-231 cells. The present study then sought to investigate the role of miR-873 
A

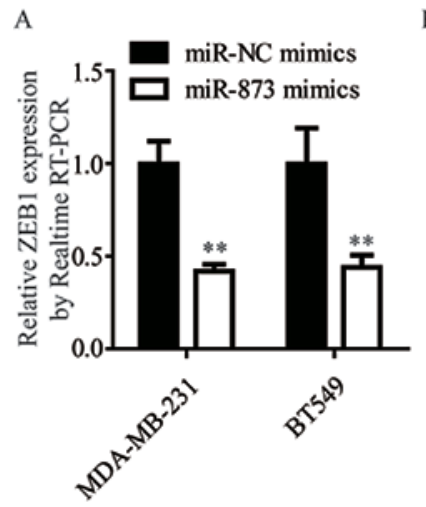

D

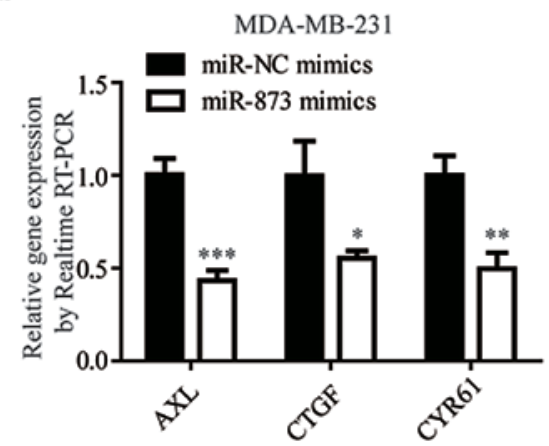

F

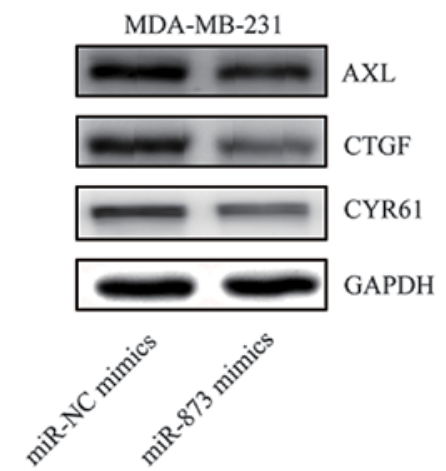

G
B

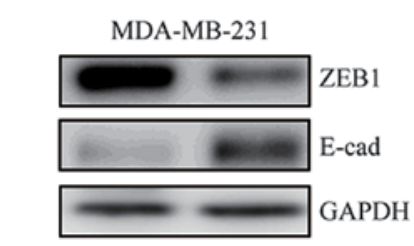

C

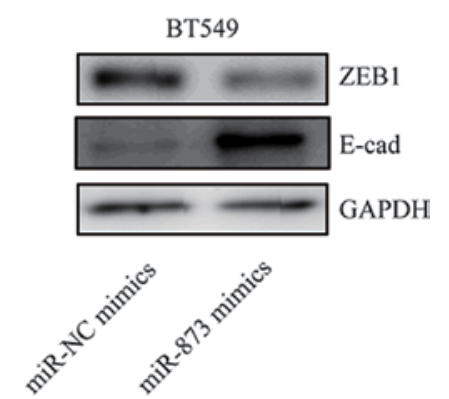

E
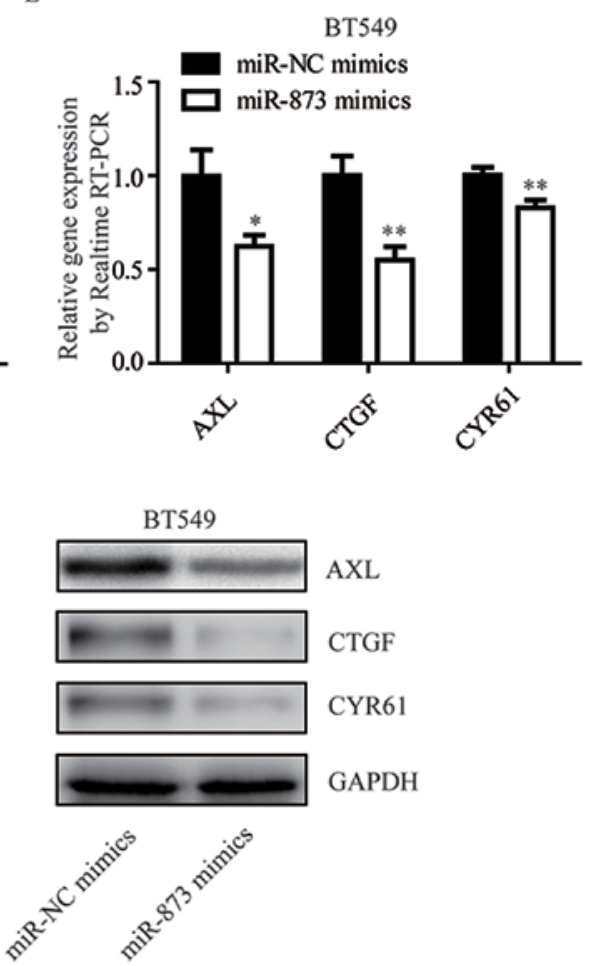

Figure 3. miR-873 represses ZEB1 expression in triple-negative breast cancer cells. (A) Overexpression of miR-873 decreased ZEB1 mRNA level in MDA-MB-231 and BT549 cells. Overexpression of miR-873 decreased ZEB1 protein level and elevated E-cadherin protein level in (B) MDA-MB-231 and (C) BT549 cells. Overexpression of miR-873 decreased AXL, CTGF and CYR61 mRNA levels in (D) MDA-MB-231 and (E) BT549 cells. Overexpression of miR-873 decreased AXL, CTGF and CYR61 protein levels in (F) MDA-MB-231 and (G) BT549 cells. ${ }^{*} \mathrm{P}<0.05,{ }^{* *} \mathrm{P}<0.01$ and ${ }^{* * * *} \mathrm{P}<0.0001$ vs. miR-NC mimics. ZEB1, zinc finger E-box binding homeobox 1; NC, negative control; AXL, AXL receptor tyrosine kinase; CTGF, connective tissue growth factor; CYR61, cysteine rich angiogenic inducer 61 ; miR, microRNA.

during the development of gemcitabine resistance using MDA-MB-231GEMr cells. Compared with parental MDA-MB-231 cells, MDA-MB-231GEMr cells were relatively insensitive towards gemcitabine treatment (Fig. 4A). Additionally, miR-873 level was significantly decreased (Fig. 4B) in MDA-MB-231GEMr cells accompanied with elevation of ZEB1 at the mRNA and protein levels (Fig. 4C and D). Consistent with the proliferation promotion role of ZEB1, the growth rate of MDA-MB-231GEMr cells was significantly increased, compared with that of MDA-MB-231 cells (Fig. 4E; $\mathrm{P}<0.05$ at $48 \mathrm{~h} ; \mathrm{P}<0.01$ at $72 \mathrm{~h}$ ).

Decrease of miR-873 contributes to gemcitabine resistance in MDA-MB-231GEMr via regulation of $Z E B 1$. In MDA-MB-231GEMr cells, the overexpression of miR-873 by transfection of miR-873 mimics sensitized cells towards gemcitabine treatment (Fig. 5A). Furthermore, silencing of ZEB1 using ZEB1 siRNAs also enhanced cell viability inhibition, which was induced by gemcitabine treatment in MDA-MB-231GEMr cells, indicating a significant reversion of gemcitabine resistance in MDA-MB-231GEMr cells (Fig. 5B and C).

\section{Discussion}

Standard chemotherapy is a major effective treatment approach for patients with TNBC that do not respond towards endocrine therapy or HER2-target therapy (23). However, almost all patients with TNBC develop chemoresistance, which eventually culminates in patient mortality (24). Determining the underlying mechanism of chemoresistance is important to improve patient outcomes. In the present study, miR-873 was 

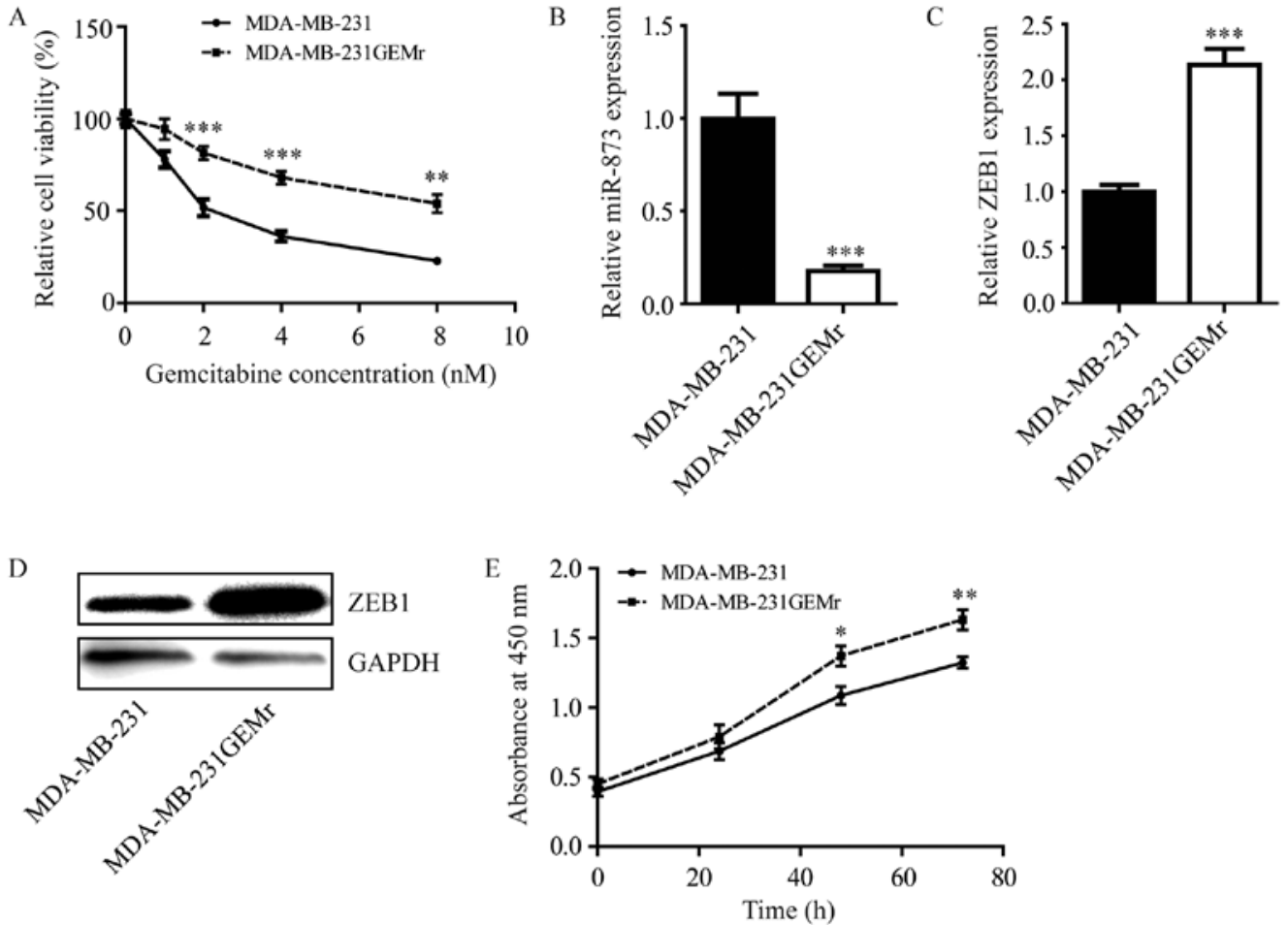

Figure 4. Altered expression of miR-873 and ZEB1 in MDA-MB-231GEMr cells. (A) Compared with MDA-MB-231, MDA-MB-231GEMr cells were relatively insensitive towards gemcitabine treatment (0-8 nM). (B) miR-873 level was decreased in MDA-MB-231GEMr cells. (C) The ZEB1 mRNA level was elevated in MDA-MB-231GEMr cells. (D) ZEB1 protein level was increased in MDA-MBA-231GEMr cells. (E) In comparison with MDA-MB-321 cells, the cell proliferation of MDA-MB-231GEMr cells was increased. ${ }^{*} \mathrm{P}<0.05,{ }^{* *} \mathrm{P}<0.01$ and ${ }^{* * *} \mathrm{P}<0.0001$ vs. MDA-MB-321 cells. ZEB1, zinc finger E-box binding homeobox 1 ; miR, microRNA.
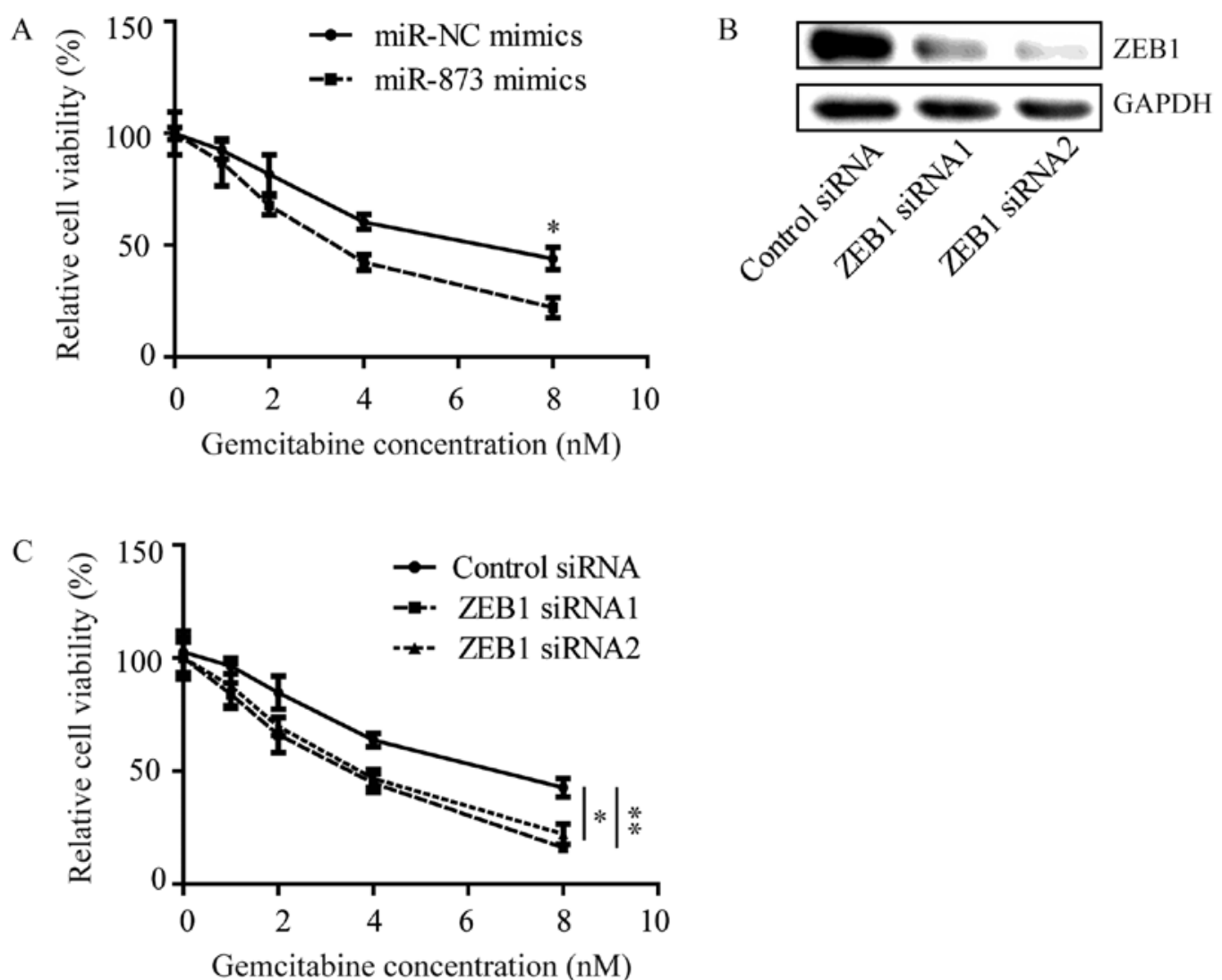

Figure 5. Overexpression of miR-873 or silencing of ZEB1 reverses gemcitabine resistance of MDA-MB-231GEMr cells. (A) Overexpression of miR-873 by transfection of miR-873 mimics reversed gemcitabine resistance of MDA-MB-231GEMr cells. "P $<0.05$ vs. miR-NC mimics. (B) ZEB1 protein level was decreased by transfection of ZEB1 siRNA1 or ZEB1 siRNA2. (C) Silencing of ZEB1 by transfection of ZEB1 siRNA reversed gemcitabine resistance of MDA-MB-231GEMr cells. ${ }^{*} \mathrm{P}<0.05 ;{ }^{* * *} \mathrm{P}<0.01$ vs. control siRNA. ZEB1, Zinc finger E-box binding homeobox 1; siRNA, small interfering RNA; NC, negative control; miR, microRNA. 
identified as a pivotal molecule in the regulation of chemotherapy sensitivity in TNBC.

Dysregulation of miRNAs was frequently observed in TNBC and contributed to the initiation, progression and chemoresistance (25). Multiple miRNAs, including miR-638 and miR-101, were decreased in TNBC tissues, compared with normal adjacent tissues, and these miRNAs could sensitize TNBC cells towards chemotherapy, while reduction of their expression contributed to chemoresistance $(26,27)$. miR-873 levels were downregulated in breast cancer tissues and tamoxifen-resistant MCF7 cells, and forced overexpression of miR-873 inhibited breast cancer cell growth and reversed tamoxifen resistance of tamoxifen-resistant MCF7 cells (11). The present study demonstrated that miR-873 regulated gemcitabine sensitivity in TNBC cells, and the transfection of MDA-MB-231 and BT549 cells with miR-873 mimics sensitized them towards gemcitabine treatment. Additionally, antagonists of miR-873 by miR-873 inhibitors weakened gemcitabine-induced cell viability inhibition in MDA-MB-231 and BT549 cells. Notably, in MDA-MB-231GEMr cells, a decreased miR-873 level, compared with their parental cells, was observed. Furthermore, silencing of miR-873 reversed gemcitabine resistance of MDA-MB-231GEMr cells. These results indicated that loss of miR-873 promotes the development of gemcitabine resistance in TNBC cells.

ZEB1 is a transcription factor well-known for its oncogenic role via inducing EMT (28), which functions in cells through various mechanisms including Wnt, nuclear factor- $\kappa$ B and miRNAs (29-31). Using TargetScan, miR-873 was predicted as a direct regulator of 3'UTR of ZEB1 mRNA. In 293 cells, miR-873 mimics greatly repressed luciferase activity of ZEB1 3'UTR-WT in a dual luciferase assay. In MDA-MB-231 cells and BT549 cells, transfection of miR-873 mimics decreased ZEB1 expression. ZEB1 could function as a transcription activator to activate YAP1 target genes, including AXL, CTGF and CYR61 expression, and as a transcription suppressor to inhibit target gene (E-cadherin) expression (19). In the present study, miR-873 overexpression increased E-cadherin expression and decreased AXL, CTGF and CYR61 expression, indicating that miR-873 could repress ZEB1 expression to regulate ZEB1 target genes levels. With self-renewal ability, cancer stem cells have been demonstrated to contribute to chemoresistance in numerous types of cancer, including breast, colon and prostate cancer, through protecting tumor cells from DNA damage and activating pathways involved in maintaining cell survival $(32,33)$. Since ZEB1 serves a pivotal role in promoting the development of cancer stem cell properties, overexpression of ZEB1 has been implicated in chemoresistance in cancer (14). The present study detected an elevation of ZEB1 in MDA-MB-231GEMr cells, indicating that ZEB1 expression was associated with chemoresistance in TNBC. Additionally, silencing of ZEB1 reversed gemcitabine resistance of MDA-MB-231GEMr cells. Collectively, the present study identified miR-873 as a novel regulator of ZEB1 3'UTR and demonstrated that miR-873 determined ZEB1 expression to alter gemcitabine sensitivity in TNBC cells.

In conclusion, the present study demonstrated that miR-873 could negatively regulate ZEB1 expression and enhance cell growth inhibition induced by treatment with gemcitabine. These data provide strong evidence that the loss of miR-873 contributes to the development of gemcitabine resistance in TNBC by controlling ZEB1 expression, which implicates miR-873 as a potential predictor and target for TNBC treatment.

\section{Acknowledgements}

Not applicable.

\section{Funding}

No funding was received.

\section{Availability of data and materials}

The datasets used and/or analyzed during the current study are available from the corresponding author on reasonable request.

\section{Authors' contributions}

GW, YD, HL, NJ, JC, AL, XT and YR designed the study and acquired the data. GW and YD established the cell line. GW, XT and YR wrote and revised the manuscript. GW supervised the study.

\section{Ethics approval and consent to participate}

Not applicable.

\section{Patient consent for publication}

Not applicable.

\section{Competing interests}

The authors declare that they have no competing interests.

\section{References}

1. Oakman C, Viale G and Di Leo A: Management of triple negative breast cancer. Breast 19: 312-321, 2010.

2. Carey LA, Dees EC, Sawyer L, Gatti L, Moore DT, Collichio F, Ollila DW, Sartor CI, Graham ML and Perou CM: The triple negative paradox: Primary tumor chemosensitivity of breast cancer subtypes. Clin Cancer Res 13: 2329-2334, 2007.

3. Papa AL, Sidiqui A, Balasubramanian SU, Sarangi S, Luchette M, Sengupta S and Harfouche R: PEGylated liposomal Gemcitabine: Insights into a potential breast cancer therapeutic. Cell Oncol (Dordr) 36: 449-457, 2013

4. Ueno H, Kiyosawa K and Kaniwa N: Pharmacogenomics of gemcitabine: Can genetic studies lead to tailor-made therapy? $\mathrm{Br}$ J Cancer 97: 145-151, 2007.

5. Samanta D, Gilkes DM, Chaturvedi P, Xiang L and Semenza GL: Hypoxia-inducible factors are required for chemotherapy resistance of breast cancer stem cells. Proc Natl Acad Sci USA 111: E5429-E5438, 2014.

6. Bartel DP: MicroRNAs: Target recognition and regulatory functions. Cell 136: 215-233, 2009.

7. Liu B, Li J and Cairns MJ: Identifying miRNAs, targets and functions. Brief Bioinform 15: 1-19, 2014.

8. Wang J, Yang M, Li Y and Han B: The role of MicroRNAs in the chemoresistance of breast cancer. Drug Dev Res 76: 368-374, 2015. 
9. Li HY, Liang JL, Kuo YL, Lee HH, Calkins MJ, Chang HT, Lin FC, Chen YC, Hsu TI, Hsiao M, et al: miR-105/93-3p promotes chemoresistance and circulating miR-105/93-3p acts as a diagnostic biomarker for triple negative breast cancer. Breast Cancer Res 19: 133, 2017.

10. Wang RJ, Li JW, Bao BH, Wu HC, Du ZH, Su JL, Zhang MH and Liang HQ: MicroRNA-873 (miRNA-873) inhibits glioblastoma tumorigenesis and metastasis by suppressing the expression of IGF2BP1. J Biol Chem 290: 8938-8948, 2015.

11. Cui J, Yang Y, Li H, Leng Y, Qian K, Huang Q, Zhang C, Lu Z, Chen J, Sun T, et al: MiR-873 regulates ER $\alpha$ transcriptional activity and tamoxifen resistance via targeting CDK3 in breast cancer cells. Oncogene 34: 4018, 2015.

12. Chen X, Zhang Y, Shi Y, Lian H, Tu H, Han S, Peng B, Liu W and He X: MiR-873 acts as a novel sensitizer of glioma cells to cisplatin by targeting Bcl-2. Int J Oncol 47: 1603-1611, 2015.

13. Wu DD, Li XS, Meng XN, Yan J and Zong ZH: MicroRNA-873 mediates multidrug resistance in ovarian cancer cells by targeting ABCB1. Tumour Biol 37: 10499-10506, 2016.

14. Zhang P, Sun Y and Ma L: ZEB1: At the crossroads of epithelial-mesenchymal transition, metastasis and therapy resistance. Cell Cycle 14: 481-487, 2015.

15. De Craene B and Berx G: Regulatory networks defining EMT during cancer initiation and progression. Nat Rev Cancer 13 97-110, 2013

16. Onder TT, Gupta PB, Mani SA, Yang J, Lander ES and Weinberg RA: Loss of E-cadherin promotes metastasis via multiple downstream transcriptional pathways. Cancer Res 68: 3645-3654, 2008.

17. Karihtala P, Auvinen P, Kauppila S, Haapasaari KM, Jukkola-Vuorinen A and Soini Y: Vimentin, zeb1 and Sip1 are up-regulated in triple-negative and basal-like breast cancers: Association with an aggressive tumour phenotype. Breast Cancer Res Treat 138: 81-90, 2013.

18. Giordano A, Gao H, Anfossi S, Cohen E, Mego M, Lee BN, Tin S, De Laurentiis M, Parker CA, Alvarez RH, et al: Epithelial-mesenchymal transition and stem cell markers in patients with HER2-positive metastatic breast cancer. Mol Cancer Ther 11: 2526-2534, 2012.

19. Lehmann W, Mossmann D, Kleemann J, Mock K, Meisinger C Brummer T, Herr R, Brabletz S, Stemmler MP and Brabletz T: ZEB1 turns into a transcriptional activator by interacting with YAP1 in aggressive cancer types. Nat Commun 7: 10498, 2016.

20. Davidson JD, Ma L, Flagella M, Geeganage S, Gelbert LM and Slapak CA: An increase in the expression of ribonucleotide reductase large subunit 1 is associated with gemcitabine resistance in non-small cell lung cancer cell lines. Cancer Res 64 3761-3766, 2004.

21. Livak KJ and Schmittgen TD: Analysis of relative gene expression data using real-time quantitative PCR and the 2(-Delta Delta C(T)) method. Methods 25: 402-408, 2001.
22. Sánchez-Tilló E, Lázaro A, Torrent R, Cuatrecasas M, Vaquero EC Castells A, Engel P and Postigo A: ZEB1 represses E-cadherin and induces an EMT by recruiting the SWI/SNF chromatin-remodeling protein BRG1. Oncogene 29: 3490-3500, 2010.

23. Podo F, Buydens LM, Degani H, Hilhorst R, Klipp E, Gribbestad IS, Van Huffel S, van Laarhoven HW, Luts J, Monleon D, et al: Triple-negative breast cancer: Present challenges and new perspectives. Mol Oncol 4: 209-229, 2010.

24. O'Reilly EA, Gubbins L, Sharma S, Tully R, Guang MH, Weiner-Gorzel K, McCaffrey J, Harrison M, Furlong F, Kell M and McCann A: The fate of chemoresistance in triple negative breast cancer (TNBC). BBA Clin 3: 257-275, 2015.

25. Yang F, Zhang W, Shen Y and Guan X: Identification of dysregulated microRNAs in triple-negative breast cancer (review). Int J Oncol 46: 927-932, 2015.

26. Liu X, Tang H, Chen J, Song C, Yang L, Liu P, Wang N, Xie X, Lin X and Xie X: MicroRNA-101 inhibits cell progression and increases paclitaxel sensitivity by suppressing MCL-1 expression in human triple-negative breast cancer. Oncotarget 6 : 20070-20083, 2015.

27. Tan X, Peng J, Fu Y, An S, Rezaei K, Tabbara S, Teal CB, Man YG, Brem RF and Fu SW: miR-638 mediated regulation of BRCA1 affects DNA repair and sensitivity to UV and cisplatin in triple-negative breast cancer. Breast Cancer Res 16: 435, 2014.

28. Aigner K, Dampier B, Descovich L, Mikula M, Sultan A, Schreiber M, Mikulits W, Brabletz T, Strand D, Obrist P, et al: The transcription factor ZEB1 (deltaEF1) promotes tumour cell dedifferentiation by repressing master regulators of epithelial polarity. Oncogene 26: 6979-6988, 2007.

29. Kahlert UD, Maciaczyk D, Doostkam S, Orr BA, Simons B Bogiel T, Reithmeier T, Prinz M, Schubert J, Niedermann G, et al: Activation of canonical WNT/ $\beta$-catenin signaling enhances in vitro motility of glioblastoma cells by activation of ZEB1 and other activators of epithelial-to-mesenchymal transition. Cancer Lett 325: 42-53, 2012.

30. Chua HL, Bhat-Nakshatri P, Clare SE, Morimiya A, Badve S and Nakshatri H: NF-kappaB represses E-cadherin expression and enhances epithelial to mesenchymal transition of mammary epithelial cells: Potential involvement of ZEB-1 and ZEB-2. Oncogene 26: 711-724, 2007.

31. Korpal M, Lee ES, Hu G and Kang Y: The miR-200 family inhibits epithelial-mesenchymal transition and cancer cell migration by direct targeting of E-cadherin transcriptional repressors ZEB1 and ZEB2. J Biol Chem 283: 14910-14914, 2008.

32. Peitzsch C, Kurth I, Kunz-Schughart L, Baumann $M$ and Dubrovska A: Discovery of the cancer stem cell related determinants of radioresistance. Radiother Oncol 108: 378-387, 2013.

33. Allegra A, Alonci A, Penna G, Innao V, Gerace D, Rotondo F and Musolino C: The cancer stem cell hypothesis: A guide to potential molecular targets. Cancer Invest 32: 470-495, 2014. 